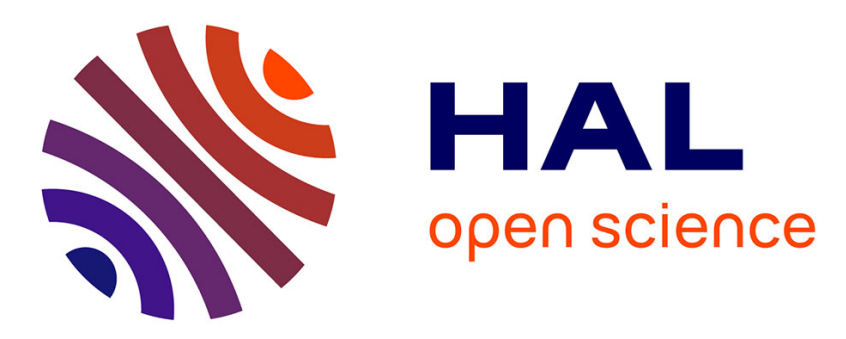

\title{
Error estimation of a neuro-fuzzy predictor for prognostic purpose.
}

Mohamed El-Koujok, Rafael Gouriveau, Noureddine Zerhouni

\section{To cite this version:}

Mohamed El-Koujok, Rafael Gouriveau, Noureddine Zerhouni. Error estimation of a neuro-fuzzy predictor for prognostic purpose.. 7th International Symposium on Fault Detection, Supervision and Safety of Technical Processes, SAFE PROCESS'09., Jun 2009, Barcelone, Spain. 6 p. hal-00402951

\section{HAL Id: hal-00402951 https://hal.science/hal-00402951}

Submitted on 8 Jul 2009

HAL is a multi-disciplinary open access archive for the deposit and dissemination of scientific research documents, whether they are published or not. The documents may come from teaching and research institutions in France or abroad, or from public or private research centers.
L'archive ouverte pluridisciplinaire HAL, est destinée au dépôt et à la diffusion de documents scientifiques de niveau recherche, publiés ou non, émanant des établissements d'enseignement et de recherche français ou étrangers, des laboratoires publics ou privés. 


\title{
Error estimation of a neuro-fuzzy predictor for prognostic purpose
}

\author{
Mohamed El-Koujok, Rafael Gouriveau, Noureddine Zerhouni \\ FEMTO-ST Institute, UMR CNRS 6174 - UFC/ENSMM/UTBM, \\ Automatic Control and Micro-Mechatronic Systems Department, \\ Besançon, France 25000 (Tel: +33-(0)381-402-801; e-mail: \\ \{Mohamed.Elkoujok, rgourive, zerhouni\}@ens2m.fr)
}

\begin{abstract}
Prognostic is recognized as a key feature as the estimation of the remaining useful life of an equipment allows avoiding inopportune maintenance spending. However, it can be difficult to implement an efficient prognostic tool since the lack of knowledge on the behavior of an equipment can impede the development of classical dependability analysis. In this context, the general purpose of the work is to define a prognostic system for which any assumption on its structure is necessary: it starts from monitoring data and goes through provisional reliability and remaining useful life by characterizing the uncertainty following from the degradation process. Developments are founded on the use of the evolving eXtended Tagaki-Sugeno system as a neurofuzzy predictor. A method to estimate the probability distribution function of the predicted degradation signal is proposed. It enables to perform a priori reliability analysis. The approach is based on a recursive calculation procedure and is thereby well adapted to online applications.
\end{abstract}

Keywords: Prognostic; prediction of degradation; confidence interval; neuro-fuzzy system.

\section{INTRODUCTION}

The high costs in maintaining complex equipments make necessary to enhance maintenance support systems and traditional concepts like preventive and corrective strategies are progressively completed by new ones like predictive and proactive maintenance (Muller et al. [2008]). Thereby, prognostic is considered as a key feature in maintenance strategies as the estimation of the provisional reliability of an equipment as well as its remaining useful life allows avoiding inopportune spending.

From the research point of view, many developments exist to support the prognostic activity (Byington et al. [2002], Jardine et al. [2006], Vachtsevanos et al. [2006]). However, in practice, choosing an efficient technique depends on classical constraints that limit the applicability of the tools: available data-knowledge-experiences, dynamic and complexity of systems, implementation requirements, available monitoring devices... Moreover, implementing an adequate tool can be a non trivial task as it can be difficult to provide effective models of dynamic systems including the inherent uncertainty of prognostic.

That said, developments of this paper are founded on the following two complementary assumptions. 1) On one hand, real systems increase in complexity and their behavior is often non-linear, which makes harder a modeling step, even impossible. Intelligent Maintenance Systems must however take it into account. 2) On the other hand, in many cases, it is not too costly to equip dynamic systems with sensors, which allows gathering real data online. Furthermore, monitoring systems evolve in this way.

According to all this, neuro-fuzzy (NF) systems appear to be very promising prognostic tools: NFs learn from examples and attempt to capture the subtle relationship among data. NFs are computationally effective techniques and are thereby well suited for practical problems, where it is easier to gather data than to formalize the behavior of the system being studied. Actual developments confirm the interest of using NFs in forecasting applications (Wang et al. [2004], Yam et al. [2001]).

In this context, the paper deals with the definition of a prognostic system for which any assumption on its structure is necessary: it starts from monitoring data and goes through provisional reliability and remaining useful life by characterizing the uncertainty following from the degradation process. More precisely, the paper emphasizes on the development of an evolving neuro-fuzzy predictor that, not only "gives" an approximation of the degradation but also associates to it a confidence measure. The model is well adapted to perform a priori reliability analysis and thereby optimize maintenance policies.

The paper is organized in four main parts. First, the concept of "prognostic" is clarified and replaced within maintenance strategies. The relationship between prognostic, prediction and online reliability is also explained: the efficiency of a prognostic system is highly dependent on its ability to perform "good" predictions as reliability indicators follow from it. This is a central point of the work. Then, the use of Takagi-Sugeno neuro-fuzzy systems for prognostic is justified and the ways of building such models are briefly discussed in the second part. An evolving neurofuzzy model for prognostic is proposed and presented. In the third part, statistical estimation techniques are adapted to the evolving neuro-fuzzy predictor in order to provide a confidence measure on prediction and thereby enable reliability analysis. Finally, the whole is illustrated with an example extracted from literature. 


\section{PROGNOSTIC AND RELIABILITY}

\subsection{From maintenance to prognostic}

Maintenance activity combines different methods, tools and techniques to reduce maintenance costs while increasing reliability, availability and security of equipments. Thus, one usually speaks about fault detection, failures diagnosis, and response development (choice and scheduling of preventive and/or corrective actions). Briefly, these steps correspond to the need, firstly, of "perceiving" phenomena, secondly, of "understanding"' them, and finally, of "acting" consequently. However, rather than understanding a phenomenon which has just appeared like a failure, it is convenient to "anticipate" its manifestation in order to take adequate actions as soon as possible. This is what can be defined as the "prognostic process".

\subsection{From prognostic to prediction}

Although there are some divergences in literature, prognostic can be defined as proposed by the International Organization for Standardization: prognostic is the estimation of time to failure and risk for one or more existing and future failure modes (ISO 13381-1 [2004]). Prognostic is also a process whose objective is to predict the remaining useful life $(R U L)$ before a failure occurs given the current machine condition and past operation profile (Jardine et al. [2006]). Two salient aspects of prognostic appear: (1) prognostic is mostly assimilated to a prediction process (a future situation must be caught), (2) prognostic is based on the failure notion, which implies that it is associated with a limit of acceptability. A central problem can be pointed out: the accuracy of a prognostic system is related to its ability to approximate and predict the degradation of an equipment; the prediction phase is a critical one.

\subsection{From prediction to reliability}

An important task of prognostic is to predict the degradation of an equipment. Following that, prognostic can also be seen as a process that allows the a priori reliability modeling. Reliability $(R(t))$ is defined as the probability that a failure does not occur before time $t$. If the random variable $\vartheta$ denotes the time of failure and $F_{\vartheta}(t)=\operatorname{Prob}(\vartheta \leq$ $t)$ is its cumulative distribution function, then:

$$
R(t)=1-F_{\vartheta}(t)
$$

Let assume now that the failure is not characterized by a random variable but by the fact that a degradation signal $(y)$ overpass a degradation limit $\left(y_{l i m}\right)$, and that this degradation signal can be estimated $(\tilde{y})$ (Fig. 1). At any time $t$, the failure probability can be expressed as follows:

$$
F(t)=\operatorname{Pr}\left[\tilde{y}(t) \geq y_{\text {lim }}\right]
$$

Let note $g(\tilde{y} / t)$ the probability density function (pdf) of the estimated degradation signal that denotes the prediction at time $t$. Thereby, by analogy with reliability theory, the reliability modeling can be expressed as follows:

$$
R(t)=1-\operatorname{Pr}\left[\tilde{y}(t) \geq y_{l i m}\right]=1-\int_{y_{l i m}}^{\infty} g(\tilde{y} / t) \cdot d y
$$

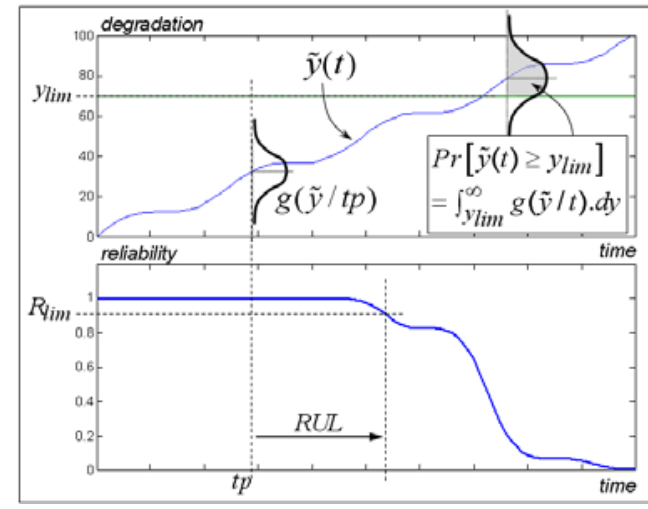

Fig. 1. Prediction and reliability modeling

The remaining useful life $(R U L)$ can finally be expressed as the time between the time in which is made the prediction $(t p)$ and the time to underpass a reliability limit $\left(R_{\text {lim }}\right)$ fixed by the practitioner (see Fig. 1). The above explanations can be generalized with a multi-dimensional degradation signal. See (Chinnam and Pundarikaksha [2004]) or (Wang and Coit [2004]) for more details.

Finally, in order to perform a priori reliability analysis and thereby evaluate the $R U L$, an effective prognostic tool should provide the pdf of the estimated degradation signal at any time $t(g(\tilde{y} / t)$ in Fig. 1). Moreover, this would enable to build confidence intervals on predictions, which can help practitioners in judging from the degradation state of the system and thereby, in taking adequate decisions.

\section{FUZZY MODELS FOR PREDICTION}

\subsection{Takagi-Sugeno system: a fitted prediction tool}

The aim of this part is not to dress an exhaustive overview of prediction techniques but to explain the orientations of works that are taken. Various prognostic approaches have been developed ranging in fidelity from simple historical failure rate models to high-fidelity physics-based models (Byington et al. [2002], Vachtsevanos et al. [2006]). Briefly, these methods can be associated with one of the following two approaches: model-based and data-driven.

Model-based methods assume that an accurate mathematical model for the analyzed system can be constructed by incorporating physical understanding of the system. However, this may also be a strong weakness: it can be difficult, even impossible to catch the system's behavior. Data-driven approaches use real data (like online gathered with sensors or operator measures) to approximate and track features revealing the degradation of components and to forecast the global behavior of a system.

Real systems are complex and their behavior is often non linear, non stationary. This consideration make harder a modeling step. Moreover, monitoring systems have evolved and it is now quite easy to online gather data. Thus, data-driven approaches are been increasingly applied to machine prognostic and recent works focus on the interest of using hybrid systems for prediction purpose. More precisely, first order Tagaki-Sugeno (TS) fuzzy models have shown improved performances over conventional approaches (Wang et al. [2004], Yam et al. [2001]). Thus, they can perform the degradation modeling step of prognostic. 


\subsection{Takagi-Sugeno models: principles}

A first order TS model is based on the fuzzy decomposition of the input space. For each part of the state space, a fuzzy rule can be constructed to make a linear approximation of the input. The global output is a combination of all rules. In others words, a TS model can be seen as a multi-model structure consisting of linear models that are not necessarily independent (Angelov and Filev [2004]). Consider Fig. 2 to explain the first order TS model. This model has two inputs variables. Two membership functions (antecedent fuzzy sets) are assigned to each one of them. The model is finally composed of two fuzzy rules. (It can be generalized to the case of $n$ inputs and $N$ rules).

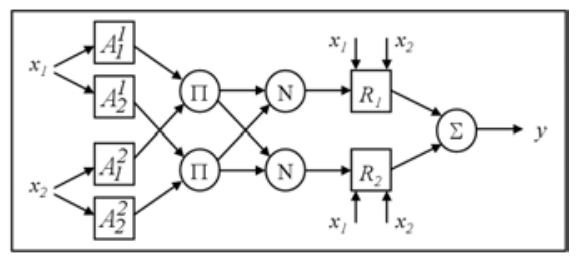

Fig. 2. First order TS model

The rules perform a linear approximation of inputs:

$$
\begin{aligned}
& R_{i}: I F x_{1} \text { is } A_{i}^{1} \text { and } \ldots \text { and } x_{n} \text { is } A_{i}^{n} \\
& T H E N y_{i}=a_{i 0}+a_{i 1} x_{1}+\ldots+a_{i n} x_{n}
\end{aligned}
$$

where $R_{i}$ is the $i^{t h}$ fuzzy rule, $N$ is the number of rules, $X=\left[x_{1}, \ldots, x_{n}\right]^{T}$ is the input vector, $A_{i}^{j}$ denotes the antecedent fuzzy sets, $j=[1, n], y_{i}$ is the output of the $i^{t h}$ linear subsystem, and $a_{i q}$ are its parameters, $q=[0, n]$. Let assume Gaussien antecedent fuzzy sets (justified by its generalization capabilities) to define the regions of fuzzy rules in which the local linear sub-models are valid:

$$
\mu_{i}^{j}=\exp ^{-\left(4\left\|x-x^{i *}\right\|_{j}\right) /\left(\left(\sigma_{i}^{j}\right)^{2}\right)}
$$

where $\sigma_{i}^{j}$ is the spread of the membership function, and $x^{i *}$ is the focal point (center) of the $i^{t h}$ rule antecedent. The firing level $\left(\tau_{i}\right)$ and the normalized firing level $\left(\lambda_{i}\right)$ of each rule are obtained as follows:

$$
\tau_{i}=\mu_{i}^{1}\left(x_{1}\right) \times \ldots \times \mu_{i}^{n}\left(x_{n}\right), \lambda_{i}=\tau_{i} / \sum_{v=1}^{N} \tau_{v}
$$

The model output is the weighted averaging of individual rules contributions. With notations, $\pi_{i}=\left[a_{i 0}, \ldots, a_{i n}\right]$ the parameters vector of the $i^{\text {th }}$ sub-model, and $x_{e}=\left[\begin{array}{ll}1 & X^{T}\end{array}\right]^{T}$ the expanded data vector, this output is expressed as:

$$
y=\sum_{i=1}^{N} \lambda_{i} y_{i}=\sum_{i=1}^{N} \lambda_{i} x_{e}^{T} \pi_{i}
$$

A TS model has two types of parameters. The non-linear parameters are those of the membership functions (the centers and spread deviations in (5)). These parameters are referred to as premise or antecedent parameters. The second types of parameters are the linear ones that form the consequent part of each rule $\left(a_{i q}\right.$ in (4)).

\subsection{Choosing a TS system: exTS for prognostic application}

Assuming that a TS model can approximate an inputoutput function (previous section), in practice, this kind of model must be tuned to fit to the studied problem. This implies two task to be performed: (1) the design of the structure (number and type of membership functions, number of rules), (2) the optimization of the parameters. For that purpose, different approaches can be used to identify a TS model: by an expert (Espinosa et al. [2005]), by using gradient descent (Jang and Sun [1995]), with genetic algorithms (Angelov and Xydeas [2006]), by clustering methods (Bezdek [1980]), or thanks to evolving algorithms (Angelov and Filev [2004], Kasabov and Song [2002]).

The choice of a TS model identification algorithm for prognostic purpose has been discussed in a previous work (ElKoujok et al. [2008]): considering the applicative restrictions that supposes the implementation of a prognostic tool, evolving TS models appear to be very promising. Firstly, they are able to update the parameters without the intervention of an expert. Secondly, they can be trained in online mode. Thirdly, they have a flexible structure that evolves as data are gathered: data are collected continuously which enables to form new rules or to modify an existing one. This last characteristics is very useful to take into account the non-stationary aspect of degradation. A particular evolving TS model is proposed by Angelov and Zhou [2006]: the evolving extended TS system (exTS).

\subsection{Learning procedure of exTS}

The learning procedure of exTS is composed of two phases: (1) an unsupervised data clustering technique is used to adjust the antecedent parameters, (2) the supervised recursive least squares learning method is used to update the consequent parameters.

\section{Clustering phase: partitioning the data space}

The exTS clustering phase processes on the global inputoutput data space: $z=\left[x^{T}, y^{T}\right]^{T}, z \in R^{n+m}$, where $n+m$ defines the dimensionality of the input/output data space. Each one of the sub-model of exTS operates in a sub-area of $z$. This clustering algorithm is based on the calculus of a potential which is the capability of a data to form a cluster (antecedent of a rule). The procedure starts from scratch and, as more data are available, the model evolves by replacement or upgrade of rules (Angelov and Zhou [2006]). This enables the adjustment of the antecedent parameters (the non-linear ones).

RLS phase: update of the consequent parameters The exTS model is used for online prediction: at prediction step $k,(7)$ can be expressed as follows:

$$
\hat{y}_{k+1}=\sum_{i=1}^{N} \lambda_{i} y_{i}=\sum_{i=1}^{N} \lambda_{i} x_{e}^{T} \pi_{i}=\psi_{k}^{T} \hat{\theta}_{k}
$$

$\psi_{k}^{T}=\left[\lambda_{1} x_{e}^{T}, \ldots, \lambda_{n} x_{n}^{T}\right]_{k}^{T}$ is the vector of the inputs weighted by normalized firing $(\lambda)$ of the rules (updated thanks to the clustering phase). $\hat{\theta}_{k}=\left[\hat{\pi}_{1}^{T}, \ldots, \hat{\pi}_{N}^{T}\right]_{k}^{T}$ is an estimation of the linear parameters of the sub-models obtained by applying the following RLS procedure:

$$
\begin{gathered}
\hat{\theta}_{k}=\hat{\theta}_{k-1}+C_{k} \psi_{k}\left(y_{k}-\psi_{k}^{T} \hat{\theta}_{k-1}\right) ; k=2,3, \ldots \\
C_{k}=C_{k-1}-\left[C_{k-1} \psi_{k} \psi_{k}^{T} C_{k-1}\right] /\left[1+\psi_{k}^{T} C_{k-1} \psi_{k}\right]
\end{gathered}
$$

whit $C_{k}$ the $R(n+1) \times R(n+1)$ co-variance matrix of parameters errors, and initial conditions $\theta_{1}=0, C_{1}=\Omega I$, where $\Omega$ is a large positive number. 


\section{INCLUDING UNCERTAINTY TO THE EXTS PREDICTION MODEL}

\subsection{Error formulation}

Unfortunately, neuro-fuzzy predictors do not provide uncertainty indicators. Thus, in this part, a way to adapt statistical estimation techniques to the evolving neurofuzzy predictor (exTS) is proposed in order to construct a confidence measure on prediction, and thereby enable reliability analysis. This is based on the a priori estimation of the error of prediction.

An exTS approximates an input-output function by optimizing non-linear and linear parameters. Yet, a modeling error must be taken into account:

$$
y_{k+1}=f\left(X_{k}, \lambda_{k}^{*}, \theta_{k}^{*}\right)+\varepsilon_{k+1} .
$$

where, $y_{k+1}$ is the real degradation situation, $X_{k}$ is the input vector at prediction step $k, \lambda_{k}^{*}$ is the optimal vector of the normalized firing level of the rules that depends on the non-linear parameters. $\theta_{k}^{*}$ is the optimal vector of the linear parameters, and $\varepsilon_{k+1}$ traduces the modeling error.

Hypothesis 1. $\varepsilon_{k+1}$ is assumed to be independently and identically distributed following a $N\left(0, s^{2}\right)$ distribution.

The prediction error $\hat{e}_{k+1}$ can be expressed as the difference between the real degradation $\left(y_{k+1}\right)$ and the output of the exTS predictor $\left(\hat{y}_{k+1}\right)$ :

$$
\hat{e}_{k+1}=y_{k+1}-\hat{y}_{k+1}=f\left(X_{k}, \lambda^{*}, \theta_{k}^{*}\right)+\varepsilon_{k+1}-\hat{y}_{k+1}
$$

Assuming that $\lambda_{k}^{*}$ is tuned by the clustering phase of the learning process, the input-ouput function $f$ is linear with regards to the consequent parameters $\theta_{k}^{*}$. According to (8), the degradation state (11) can be expressed as follows:

$$
\begin{aligned}
y_{k+1} & =f\left(X_{k}, \lambda_{k}^{*}, \theta_{k}^{*}\right)+\varepsilon_{k+1} \\
& =\psi_{k}^{T} \theta_{k}^{*}+\varepsilon_{k+1}=f^{*}+\varepsilon_{k+1}
\end{aligned}
$$

where $f^{*}=\psi_{k}^{T} \theta_{k}^{*}$ is introduced for demonstration. According to (8) and (13), the error of prediction (12) can finally be expressed as:

$$
\hat{e}_{k+1}=\psi_{k}^{T} \theta_{k}^{*}+\varepsilon_{k+1}-\psi_{k}^{T} \hat{\theta}_{k}
$$

\subsection{Error estimation}

Next paragraphs present a way to estimate the error of prediction by calculating a priori the mean and variance of the error expressed in (14).

Mean of the error estimation. The mean of $\hat{e}_{k+1}$ is:

$$
\begin{aligned}
\mu_{\hat{e}_{k+1}} & =\mu_{\left[y_{k+1}\right]}-\mu_{\left[\hat{y}_{k+1}\right]}=\mu_{\left[f^{*}+\varepsilon_{k+1}\right]}-\mu_{\left[\hat{y}_{k+1}\right]} \\
& =\mu_{\left[f^{*}\right]}+\mu_{\left[\varepsilon_{k+1}\right]}-\mu_{\left[\hat{y}_{k+1}\right]}
\end{aligned}
$$

According to hypothesis 1 , the mean of $\varepsilon_{k+1}$ is zero. Thus, assuming that $\theta_{k}^{*}$ and $\psi_{k}$ are not random variables,

$$
\begin{aligned}
\mu_{\hat{e}_{k+1}} & =\mu_{\left[\psi_{k}^{T} \theta_{k}^{*}\right]}-\mu_{\left[\psi_{k}^{T} \hat{\theta}_{k}\right]}=\psi_{k}^{T} \mu_{\left[\theta_{k}^{*}\right]}-\psi_{k}^{T} \mu_{\left[\hat{\theta}_{k}\right]} \\
& =\psi_{k}^{T} \theta_{k}^{*}-\psi_{k}^{T} \mu_{\left[\hat{\theta}_{k}\right]}
\end{aligned}
$$

And, the mean of $\hat{\theta}_{k}$ is $\theta_{k}^{*}$, so $\mu_{\hat{e}_{k+1}}=0$. The exTS model is an unbiased predictor.
Variance of the error estimation. The variance of the error $\hat{e}_{k+1}$ is:

$$
\begin{aligned}
\sigma_{\hat{e}_{k+1}}^{2} & =\sigma_{\left[f^{*}+\varepsilon_{k+1}-\hat{y}_{k+1}\right]}^{2} \\
& =\sigma_{\left[f^{*}+\varepsilon_{k+1}\right]}^{2}+\sigma_{\left[\hat{y}_{k+1}\right]}^{2}-2 \operatorname{Cov}_{\left[f^{*}+\varepsilon_{k+1}, \hat{y}_{k+1}\right]}
\end{aligned}
$$

Assuming that $f^{*}$ is not a random variable, and that $\hat{y}_{k+1}$ and $\varepsilon_{k+1}$ are uncorrelated,

$$
\begin{aligned}
\sigma_{\hat{e}_{k+1}}^{2} & =\sigma_{\left[\varepsilon_{k+1}\right]}^{2}+\sigma_{\left[\hat{y}_{k+1}\right]}^{2}-2 \operatorname{Cov}\left(\varepsilon_{k+1}, \hat{y}_{k+1}\right) \\
& =\sigma_{\left[\varepsilon_{k+1}\right]}^{2}+\sigma_{\left[\hat{y}_{k+1}\right]}^{2}=\sigma_{\left[\varepsilon_{k+1}\right]}^{2}+\sigma_{\left[\psi_{k}^{T} \hat{\theta}_{k}\right]}^{2} \\
& =\sigma_{\left[\varepsilon_{k+1}\right]}^{2}+\psi_{k}^{T} \sigma_{\left[\hat{\theta}_{k}\right]}^{2} \psi_{k}
\end{aligned}
$$

$\sigma_{\left[\hat{\theta}_{k}\right]}^{2}$ is the variance of the parameters estimation, thus, according to (10),

$$
\begin{aligned}
\sigma_{\hat{e}_{k+1}}^{2} & =\sigma_{\left[\varepsilon_{k+1}\right]}^{2}+\psi_{k}^{T} C_{k} \psi_{k} \\
& =s^{2}+\psi_{k}^{T}\left(C_{k-1}-\frac{C_{k-1} \psi_{k} \psi_{k}^{T} C_{k-1}}{1+\psi_{k}^{T} C_{k-1} \psi_{k}}\right) \psi_{k}
\end{aligned}
$$

Finally, the error of prediction can be represented by a normal distribution as follows:

$$
\hat{e}_{k+1}: N\left[0, s^{2}+\psi_{k}^{T} C_{k} \psi_{k}\right]
$$

\subsection{Construction of a prediction interval}

According to (12), $y_{k+1}=\hat{y}_{k+1}+\hat{e}_{k+1}$. Thus, thanks to (20), a confidence interval of the output of the prediction system can be constructed.

Case 1. If in hypothesis $1, \sigma_{\left[\varepsilon_{k+1}\right]}^{2}=s^{2}$ is supposed known, then the interval of prediction is:

$$
y_{k+1} \in\left[\psi_{k}^{T} \hat{\theta}_{k} \pm \phi_{\frac{\alpha}{2}} \cdot \sqrt{\sigma_{\left[\varepsilon_{k+1}\right]}^{2}+\psi_{k}^{T} \cdot C_{k} \cdot \psi_{k}}\right]
$$

where $\phi_{\frac{\alpha}{2}}$ is the inverse of the normal cumulative distribution function for the confidence bound $\alpha$.

Case 2. If in hypothesis $1, \sigma_{\left[\varepsilon_{k+1}\right]}^{2}=s^{2}$ is supposed unknown, then it must be estimated:

$$
\hat{\sigma}_{\left[\varepsilon_{k+1}\right]}^{2}=\left[\sum_{i=1}^{k} y_{k}-\hat{y}_{k}\right] /[k-p]
$$

where $p=(n+1) \times N$ the number of parameters of $\hat{\theta}_{k}$.

Following that, an interval of prediction is given by:

$$
y_{k+1} \in\left[\psi_{k}^{T} \hat{\theta}_{k} \pm t_{k-p}\left(1-\frac{\alpha}{2}\right) \sqrt{\hat{\sigma}_{\left[\varepsilon_{k+1}\right]}^{2}+\psi_{k}^{T} C_{k} \psi_{k}}\right]
$$

where $t_{k-p}\left(1-\frac{\alpha}{2}\right)$ is a $t$ distribution with $k-p$ degrees of freedom and $100\left(1-\frac{\alpha}{2}\right)$ is the percentile with confidence bound $\alpha$.

\subsection{Estimation of the output with a pdf}

As mentioned in the last paragraph of section 2.3, in order to perform a priori reliability analysis and thereby evaluate the $R U L$, an effective prognostic tool should 
provide the pdf of the estimated degradation signal.

The degradation signal estimation can be expressed as:

$$
\tilde{y}_{k+1}=\hat{y}_{k+1}+\varepsilon_{k+1}
$$

Assuming that at each step $k$, the next prediction $\left(\hat{y}_{k+1}\right)$ can be viewed as centered in itself, and according to (18), the prediction can be represented by a normal distribution: $\hat{y}_{k+1}: N\left(\hat{y}_{k+1}, \psi_{k}^{T} \cdot C_{k} \cdot \psi_{k}\right)$. Thereby, assuming that $\hat{y}_{k+1}$ and $\varepsilon_{k+1}$ are not correlated, $\tilde{y}_{k+1}$ is also normally distributed: $N\left(\mu_{\left[\hat{y}_{k+1}\right]}+\mu_{\left[\varepsilon_{k+1}\right]}, \sigma_{\left[\varepsilon_{k+1}\right]}^{2}+\sigma_{\left[\hat{y}_{k+1}\right]}^{2}\right)$.

Finally, the developed method enables to give the probability density function of the estimation of the real output that is being predicted:

$$
\tilde{y}_{k+1}: N\left(\hat{y}_{k+1}, \sigma_{\left[\varepsilon_{k+1}\right]}^{2}+\psi_{k}^{T} \cdot C_{k} \cdot \psi_{k}\right)
$$

\section{ILLUSTRATION}

\subsection{Object: predict a degradation and estimate the RUL}

The aim of this part is to illustrate the whole proposition: estimate the remaining useful life of an equipment by predicting its degradation state in time with an exTS system, and by including the uncertainty following from this prediction phase in order to perform a priori reliability modeling. The simulations are based on the prediction of a real degradation phenomenon.

The simulated degradation is a first-order chemical reaction introduced by (Meeker and LuValle [1995]). It describes the growth of failure-causing conducting filaments of chlorine-copper compounds in printed-circuit boards. In this model, $\mathrm{CH}_{1}$ is the amount of chlorine available for reaction and $\mathrm{CH}_{2}$ is the proportional to the amount of failure-causing chlorine-copper compounds at time $t$. Under appropriate conditions of temperature, humidity, and electrical charge, there is a chemical reaction in which copper combines with chlorine $\left(\mathrm{CH}_{1}\right)$ to produce $\left(\mathrm{CH}_{2}\right)$ with a constant degradation rate $r_{1}$ :

$$
C H_{2}(t)=C H_{1}(0)\left[1-\exp \left(-r_{1} . t\right)\right]
$$

where $C H_{1}(0)$ is the initial amounts of chlorine available for reaction.

\subsection{Construction of the degradation signal}

In this paper, the proposed approach to perform prognostic assumes that no understanding of the degradation phenomena is necessary as the exTS predictor would track it. Thus, the mathematical expression of (26) was not used as a model but to generate realistic simulation data: this was done by adding a random value (uniform distribution) as a noise to the calculated value of $\left(\mathrm{CH}_{2}\right)$. Fig. (3) gives an illustration of both signals with the following parametrization of the model: $C H_{1}(0)=100$ and $r_{1}=0.005$.

\subsection{Simulation conditions}

Following the precedent procedure (section 5.2), 1000 degradation data samples were generated. Let name them from $\mathrm{CH}_{2}(0)$ to $\mathrm{CH}_{2}(999)$. The first 150 samples were used to train the predictive model, whereas the remaining ones to test it, construct a prediction interval and apply the proposed methodology for the estimation of the $R U L$.

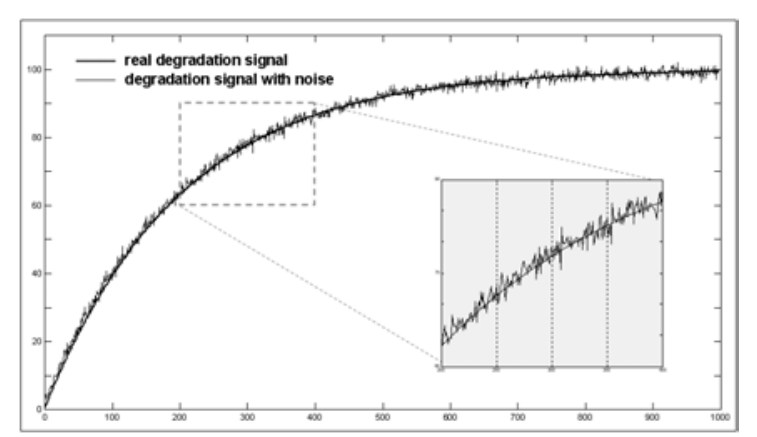

Fig. 3. Degradation signals

Four input variables related to the amount of chlorinecopper compounds were used to make a one step-ahead prediction. When ending the learning phase, multi step ahead predictions were made by injecting the previous predictions into the model. Assuming that $t$ denotes the current time, the exTS model was built as summarized in Table 1 . Note that in this example, only the past values of the $\mathrm{CH}_{2}$ are used to track the degradation, but it may be possible to add another inputs (temperature, humidity, etc.) to ameliorate the prediction performances.

\begin{tabular}{|l|l|l|l|}
\hline \multicolumn{2}{|l|}{} & $\begin{array}{l}\text { Learning } \\
t=3 \ldots 149\end{array}$ & $\begin{array}{l}\text { Test } \\
t=150 \ldots 999\end{array}$ \\
\hline Inputs & $x_{1}$ & $C H_{2}(t)$ & $\hat{C} H_{2}(t)$ \\
& $x_{2}$ & $C H_{2}(t-1)$ & $\hat{C} H_{2}(t-1)$ \\
& $x_{3}$ & $C H_{2}(t-2)$ & $\hat{C} H_{2}(t-2)$ \\
& $x_{4}$ & $C H_{2}(t-3)$ & $\hat{C} H_{2}(t-3)$ \\
\hline Output & $\hat{y}_{t+1}$ & $\hat{C} H_{2}(t+1)$ & $\hat{C} H_{2}(t+1)$ \\
\hline
\end{tabular}

Table 1. Prediction model

\subsection{Results and discussion}

In order to perform reliability modeling and $R U L$ estimation to the case studied (chemical degradation process simulation), the following decision criteria were chosen:

(1) a $95 \%$ confidence interval for predictions,

(2) a degradation limit $\left(y_{\text {lim }}\right)$ of $85 \%$ of the total amount of failure-causing chlorine-copper compounds $\mathrm{CH}_{2}$,

(3) a reliability limit $R_{\text {lim }}=0.8$ to estimate the $R U L$.

Simulation results are reported in Fig. 4. The superior part illustrates the procedure to forecast the degradation phenomenon including a prediction confidence interval (section 4.3). Even if the learning process was stopped at time $t=150$, the predicted values are very closed to the real ones. (That illustrates also the accuracy of the exTS predictor). The inferior part of Fig. 4 shows the reliability modeling and $R U L$ estimation by the methodology proposed in this paper (sections 2.3 and 4.4). Thanks to the prediction interval and to the degradation limit, one can bound the interval in which the failure should occur. Moreover, if the practitioner is able to fix a reliability limit, it is quite easy to extract the remaining useful life of the system. All these indicators should help practitioners in choosing adequate maintenance policies. From the applicative point of view, note that all calculus are made 
in a recursive manner which ensure good computational performances.

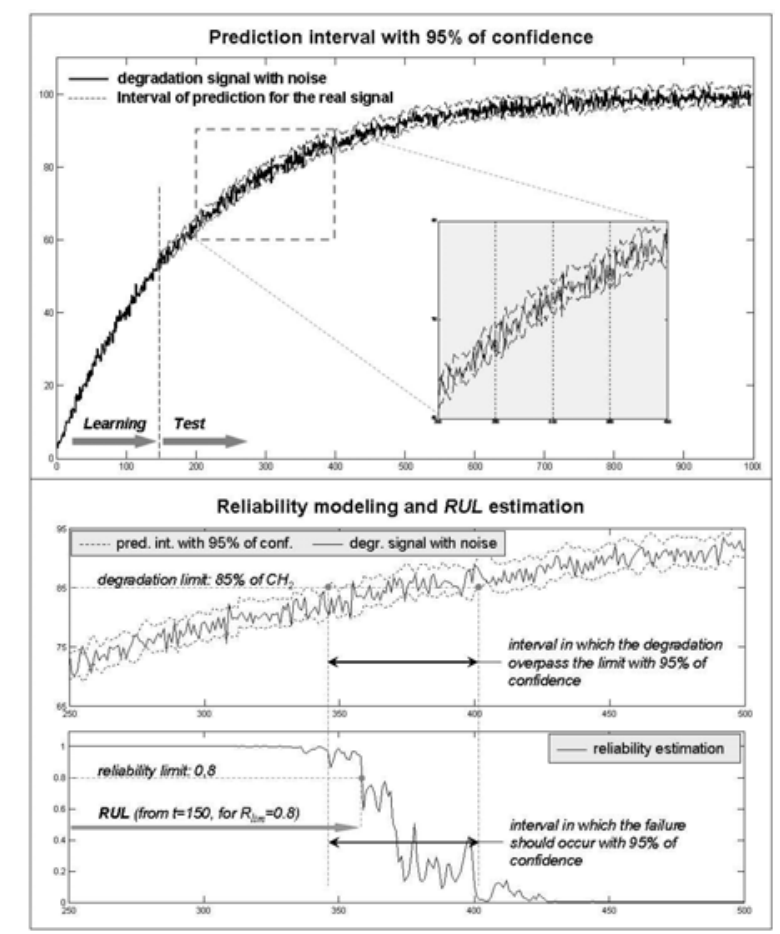

Fig. 4. Simulation results

\section{CONCLUSION AND WORKS IN PROGRESS}

In maintenance field, prognostic is recognized as a key feature as the estimation of the remaining useful life of an equipment allows avoiding inopportune spending. However, it can be difficult to define and implement an adequate and efficient prognostic tool that includes the inherent uncertainty of the prognostic process. Indeed, an important task of prognostic is that of prediction since prognostic can also be seen as a process that allows the reliability modeling. In this context, the paper deals with the definition of a prognostic system for which any assumption on its structure is necessary: it starts from monitoring data and goes through provisional reliability and remaining useful life by characterizing the uncertainty following from the degradation process. The paper emphasizes on the use of the evolving neuro-fuzzy predictor exTS. A method to associate a confidence measure to the prediction is proposed and illustrated. This procedure is based on the adaptation of statistical techniques. The model is well adapted to perform a priori reliability analysis since it provides the pdf of prediction.

Developments are at present extended in two principal ways. Firstly, the prediction performances are been studied in a more systematical way: by studying the learning convergence conditions and the impact of non-stationary noise of inputs signals. Secondly, developments are led in order to ensure a confidence level by modifying the learning algorithms. The underlying idea is that practitioners surly prefer a "well known constant" prediction error than a "sometimes catastrophic ones". This work is led with the objective of being integrated to the e-maintenance platform of a French industrial partner (em@systec).

\section{REFERENCES}

P. Angelov and D. Filev. An approach to online identification of takagi-sugeno fuzzy models. IEEE Trans. Syst. Man Cybern. - Part B: Cybernetics, 34:484-498, 2004.

P. Angelov and C. Xydeas. Fuzzy systems design: direct and indirect approaches. Soft Comp., 10:836-849, 2006.

P. Angelov and X. Zhou. Evolving fuzzy systems from data streams in real-time. In Proc. of the Int. Symp. on Evolving Fuzzy Systems, UK, pages 26-32, 2006.

J. Bezdek. A convergence theorem for the fuzzy isodata clustering algorithms. IEEE Tran. Pattern Analysis Machine Intell., 1-2:1-8, 1980.

C. Byington, M. Roemer, G. Kacprzynski, and T. Galie. Prognostic enhancements to diagnostic systems for improved condition-based maintenance. IEEE Aerospace Conference, Big Sky, USA, 2002.

R. Chinnam and B. Pundarikaksha. A neurofuzzy approach for estimating mean residual life in condtitionbased maintenace systems. Int. J. Materials and Product Technology, 20:166-179, 2004.

M. El-Koujok, R. Gouriveau, and N. Zerhouni. Development of a prognostic tool to perform reliability analysis. In Proc. of the ESREL - 17th SRA-Europe Conf., Valencia, Spain, sept. 22-25, volume 1, pages 191-199, 2008.

J. Espinosa, J. Vandewalle, and V. Wertz. Fuzzy Logic, Identification and Predictive Control (Advances in Ind. Control). Springer-Verlag, U.S.A., 2005.

ISO 13381-1. Condition monitoring and diagnostics of machines - prognostics - Part1: General guidelines. Int. Standard, ISO, 2004.

J. Jang and C. Sun. Neuro-fuzzy modeling and control. IEEE Proceedings, 83:378-406, 1995.

A. Jardine, D. Lin, and D. Banjevic. A review on machinery diagnostics and prognostics implementing condition-based maintenance. Mech. Syst. and Sign. Proc., 20:1483-1510, 2006.

N. Kasabov and Q. Song. Denfis: Dynamic evolving neural-fuzzy inference system and its application for time-series prediction. IEEE Transaction on Fuzzy Systems, 10-2:144-154, 2002.

W.Q. Meeker and M.J LuValle. An accelerated life test model based on reliability kinetics. Technometrics, 372:133-146, 1995.

A. Muller, M.C. Suhner, and B. Iung. Formalisation of a new prognosis model for supporting proactive maintenance implementation on industrial sytem. Reliability Engineering and System Safety, 93:234-253, 2008.

G. Vachtsevanos, F.L. Lewis, M. Roemer, A. Hess, and B. Wu. Intelligent Fault Diagnosis and Prognosis for Engineering Systems. Wiley \& Sons, New Jersey, 2006.

P. Wang and D. Coit. Reliability prediction based on degradation modeling for systems with multiple degradation measures. Proc. of Reliab. and Maintain. Ann. Symp. - RAMS, pages 302-307, 2004.

W. Wang, M.F. Goldnaraghi, and F. Ismail. Prognosis of machine health condition using neurofuzzy systems. Mech. Systems and Signal Proc., 18:813-831, 2004.

R.C.M. Yam, P.W. Tse, L. Li, and P. Tu. Intelligent predictive decision support system for condition-based maintenance. International Journal of Advanced Manufacturing Technology, 17:383-391, 2001. 